\title{
Reply to commentary by G. Sikri and S. Dua on the article "Correlation between single nucleotide polymorphisms in hypoxia-related genes and susceptibility to acute high-altitude pulmonary edema" published in Genetics and Molecular Research 14 (3): 11562-11572 to the letter published in Genet. Mol. Res. 14 (4): 15904-15905
}

L.J. Wu

Research and Service Center of Laboratory Medicine, Chengdu Military General Hospital, Chengdu, Sichuan, China

Corresponding author: L.J. Wu

E-mail: 379197283@qq.com

Genet. Mol. Res. 15 (1): gmr.15016155

Received January 24, 2016

Accepted January 24, 2016

Published March 24, 2016

DOI http://dx.doi.org/10.4238/gmr.15016155

The object in this study was a Han Chinese population in Lhasa, with $3658 \mathrm{~m}$ in altitude from Chengdu, which has $505 \mathrm{~m}$ in altitude by air. Within 24 to $48 \mathrm{~h}$ before the subjects arrived in the plateau, they completed a basic situation questionnaire, under the guidance of medical staff. Within 24 to $48 \mathrm{~h}$ after they reached the plateau, the subjects completed an acute plateau disease questionnaire. The diagnostic standard of HAPE and the diagnosis of acute plateau disease were adopted by the Lake Louise diagnostic standards in 1991 and the Chinese Medical Association promulgated the domestic diagnostic criteria on the Third National Plateau Medical 
Academic Seminar. Other diseases that cause acute symptoms of altitude sickness, such as plateau pulmonary edema, plateau cerebral edema, acute respiratory infections and neurosis, were excluded. According to the Lake Louise standard, questionnaire and symptom score values $>3$ points were diagnosed as HAPE. According to the national standard, the questionnaire and symptom score values $>5$ points were diagnosed as HAPE.

At the present, morbidity of HAPE remains relatively high in China. The research shows that usually in the case of not taking preventive measures, according to the factors such as altitude, speed, and time of arrival, the incidence of HAPE is 30 to $90 \%$. Epidemiological survey shows that HAPE happens at an altitude of $2500 \mathrm{~m}$ above the plateau. And an altitude of $3000 \mathrm{~m}$ above the plateau of China is occupied $1 / 6$ of the total land area, the population of permanent residents is more than sixty million. We detected the 200 cases who adapted to plateau and the 106 cases of patients with HAPE susceptibility gene SNP locus, and got the key SNP loci of HAPE susceptibility genes. We made the corresponding gene chip diagnostic kits. 